\title{
Correspondence
}

\section{Quantitative audit of the content of histopathology reports}

Drs Campbell and Griffiths give an account of how histopathology reports can be produced at a uniformly high standard that is a tribute to the working practice in their department. ${ }^{1}$ In laboratories in which computer systems permit the use of "canned text", the initiative could be extended and

\section{Clinical history}

\section{Macroscopical}

This specimen consists of solid / friable fragments of grey tissue measuring up to ${ }^{\star} \mathrm{mm}$ and totalling ${ }^{\star} \mathrm{ml}$, ${ }^{\star} \mathrm{mg}$.

\section{Microscopical}

These are fragments of a well / moderately / poorly differentiated, transitional cell carcinoma of bladder showing a solid / papillary / inverted growth pattern. There is evidence of invasion of the submucosa / and muscle coat in * our of * fragments.

There is no adjacent flat urothelium present. / The adjacent flat urothelium present shows in situ malignant / dysplastic change which increases the risk of recurrence or further tumours.

Invasive transitional cell carcinoma of bladder

(grade $1 / 2 / 3$, stage $1 \mathrm{~b} / 2$ at least)

SNOMED CODES

T74000 URINARY BLADDER

M81203 CARCINOMA, TRANSITIONAL CELI

The content of the template report is that agreed as the minimum for the specimen type and would be based on the guidelines described by Campbell and Griffiths. Asterisks and slashes are used to indicate where a mandatory addition or deletion should be made. Clearly the pathologist must have the option of adding to or deleting from the template. $\mathrm{He}$ or she will, however, be confident that they have not omitted an important part of the report.

We have used this approach for nearly three years; a directory of over 4000 standard reports covering much of general histopathology and cytology has been developed. These are recalled using intuitive file names (such as "BLTCC" for the above report) or text indexing and retrieval software. As we can generate appropriate and complete reports bearing accurate SNOMED codes without the delay of an office transcription stage, the mean reporting time has been reduced from 3.95 days to 2.63 days (based on audit of 600 casemix matched specimens). The quality of our reports has been enhanced. Because the diagnostic criteria for rarer diagnoses are included in the template, there is also the potential for greater diagnostic accuracy, as the pathologists has to read and agree to these criteria when he or she edits the template. Our coverage of some diagnostic areas is relatively comprehensive. Therefore, the use of standard histopathological terms or an immunohistochemical profile as key words to search the directory will select the report giving the correct diagnosis or a series of reports representing a differential diagnosis. We therefore have the beginnings of a "textpert" system.

Pathologists who believe that every report must be individually hand crafted without consolidated by the provision of template reports with variable sections which can be eleted.

Campbell and Griffiths' paper, a temp as a starting point for the reporting of a bladder biopsy specimen containing invaive transitional cell carcinoma might be as follows: such aids or who never intend to use computer will not take to this idea. However, those who cooperate to standardise their service, like Drs Campbell and Griffiths and their colleagues, might like to consider how "canned text" could be used to improve or at least standardise histopathology and cytology reports. Clearly the work required to develop such tools need not be duplicated in every laboratory. Pathologists who consider themselves to have a special interest in any sector of diagnostic histopathology or cytology and who would like to contribute to our report template project are invited to contact the author.

\section{SB COGHILL Department of Cellular Pathology, Northampton General}

1 Campbell F, Griffiths DFR. Quantitative audit of the content of histopathology audit of the content of histopath
reports. I Clin Pathol 1994;47:360-1.

Estimation of haemoglobin concentrations using spectrophotometric tests

Dr Goodrick and colleagues recently reported a patient with a monomeric IgM paraprotein whose haemoglobin estimations were spuriously raised. This was apparently due to interference with the spectrophotometric analytical method by the formation of an optically dense precipitate of plasma and the Coulter lysing agent. A smiliar mechanism has been shown in some of the occasional reports of pseudohyperphosphataemia in patients with monoclonal gammopathies, including Waldenström's macroglobulinaemia. Lipaemia is also recognised to confound the photometric techniques, ${ }^{3}$ as happens with haemoglobin.

Tokmakiian and colleagues reported a patient with an IgM $\kappa$ monoclonal gammopathy with spurious hypoglycaemia and hypophosphataemia. ${ }^{4}$ These phenomena were eventually shown to be due to excessive sample blankings in the automated chemical analyser due to precipitation of IgM monoclonal protein in distilled water which was used as the serum diluent.

Instrument printouts also showed an haemolysis "flag" despite the absence of visible haemolysis or turbidity. In the same way that this patient's spurious hypophosphataemia resulted from a reversal of the mechanism underlying most cases of pseudohyperphosphataemia, ${ }^{2}$ so the spurious haemolysis was probably the converse of the phenomenon described by Goodrick and colleagues.

These findings suggest that particular care should be exercised when assessing any laboratory variable estimated by spectrophotometric tests in patients with monoclonal gammopathies. Lai and colleagues, for instance, found unsuspected pseudohyperphosphataemia in 11 of 41 patients with multiple myloma. ${ }^{5}$ Without the clinical acumen shown by Goodrick and colleagues, many similar but less pronounced examples of these phenomena might have been inadvertently overlooked. Department of Anatomy, University of Cambridge, Downing Street, Cambridge CB2 $3 D Y$

1 Goodrick MJ, Boon RJ, Bishop RJD, Coplestone JA, Prentice AG. Inaccurate haemoglobin estimation in Waldenström's macroglobulinaemia: unusual reaction with monomeric IgM paraprotein. 7 Clin Pathol 1993;46:1138-9.

\section{Capnocytophaga canimorsus in} peripheral blood smears

Fife et al suggest that the initial morphology and staining characteristics of organisms seen in the peripheral blood film of septicaemic patients may be useful for provisional identification and also in the choice of initial treatment. ${ }^{1}$ The following two points are relevant to the discussion.

Firstly, the possibility of microbial contamination of staining reagents, slide or full blood count container, must be borne in mind. And where findings would be considered unusual in the particular clinical setting, due caution in interpretation should be observed.

Secondly, the authors did not mention Capnocytophaga canimorsus (dysgonic fermenter type 2) septicaemia, in which Gram staining of peripheral blood is of confirmed worth. $C$ canimorsus septicaemia is particularly associated with patients whose spleens have been removed, following animal bites 
brief descriptions of geographical distribution, morphology, and life cycle.

Section 2 contains over 250 colour photographs covering a wide range of parasite morphology, pathology (including stained sections), and clinical pictures with captions on the facing page. Although the overall quality of the photographs is excellent, I was disappointed to see a lack of size markers on all but a handful. In the clinical laboratory size is of vital importance for identifying ova and cysts.

The third section contains black and white electron micrographs, radiographs, and other illustrations, separated from the colour section for reasons of economy. This does not detract from the atlas in any way, and indeed some of the scanning electron microscopic images are quite breathtaking. I would, however, like to have seen some indication of size on the photographs.

This atlas has a spacious and orderly feel to it, and I am impressed by the overall quality. Clinical microbiologists, particularly those in training, will find it useful.

Atlas of Ovarian Tumors. L Deligdisch, A Altchek, CJ Cohen. (Pp 182; £94.) Igaku-Shoin. 1994. ISBN 0-89640-240-1.

This sumptuously produced atlas is subdivided into two main sections with three chapters devoted to "clinical aspects" and seven chapters allocated to "pathology". This strategy may be convenient in a multiauthor text, but it has resulted in a clinical section which is pathologically naive and a pathological section impoverished by the paucity of clinicopathological correlation.

The wide-ranging introductory chapter, which covers epidemiology, genetics, molecular biology, early diagnosis, and screening for ovarian cancer, provides a useful overview of the subject, although the emphasis placed on ultrasonography is excessive. The two ensuing chapters, both rather lengthy and repetitive, are devoted to management of ovarian carcinoma and nonepithelial tumours, respectively.

The pathology section comprises four chapters devoted to primary epithelial neoplasms including a whole chapter on the interesting but controversial subject of ovarian intraepithelial neoplasia. Other chapters deal with sex cord-stromal tumours, germ cell tumours, and metastatic tumours. This section is well illustrated with adequate photomicrographs and gross photographs of excellent quality. However, many entities are skimpily and uncritically described with no attempt to evaluate the taxonomic overenthusiasm displayed by recent authors in this field. On the other hand, many rare but well established entities are not included. An even more serious drawback for a book aimed at the practising histopathologist is the lack of consideration given to possible differential diagnoses.

In conclusion, this new atlas is unlikely to fulfil the need for a comprehensive, authoritative, and up to date reference text on ovarian neoplasms. It cannot be recommended as a bench book for the reporting room.

\section{Notices}

\section{Postgraduate course: \\ Current concepts in surgical pathology}

November 14-18 1994

\section{Massachusetts General Hospital,} Harvard Medical School

This course is designed for pathologists at resident and practitioner levels. It will provide an in-depth review of diagnostic surgical pathology with emphasis on morphological features, newly recognised entities, and new techniques, presented by the faculty of the Department of Pathology, Massachusetts General Hospital. Instruction will be primarily by lecture, but will also include discussion periods. Each participant will receive a comprehensive course syllabus.

The course has category 1 accreditation for about 35 hours CME credit by the American Medical Association. The fee for the course is $\$ 785.00$ (residents and fellows $\$ 575 \cdot 00$ ).

For further information contact: Department of Continuing Education, Harvard Medical School, 25 Shattuck Street, Boston, MA 02115 USA (Tel: 0101 (617) 432 1525).

\section{Update on Cerebrovascular Pathology}

Thursday 8 December 1994 (one-day) to be held at The Royal College of Pathologists, 2 Carlton House Terrace, London SW1Y 5AF.

The meeting is open to members and non-members of the College. Further details and application forms can be obtained from the Scientific Meetings Officer, RCPath, 2 Carlton House Terrace, London SW1Y 5AF (Tel: 0719305862 ext: 24/26).

\section{Cytopathology for histopathologists} Northwick Park Hospital

30 January-3 February 1995

This is an intensive course in cytopathology suitable for candidates preparing for the MRCPath examination in histopathology, and for established histopathologists requiring revision. It is given by the Department of Cellular Pathology, Northwick Park Hospital (Dr Elizabeth A Hudson) and the Department of Cytopathology, St Mary's Hospital Medical School, University of London (Professor Dulcie Coleman).

The programme will consist of lectures, microscopy sessions, and discussions. Topics will include cytopathology of the cervix, urine, the respiratory tract, serous effusions and fine needle aspiration cytology of breast and other sites. The course is limited to 30 participants. The course fee is $£ 300$ excluding accommodation.

Applications and enquiries should be made to: Dr Elizabeth Hudson, Department of Cellular Pathology, Northwick Park Hospital, Harrow, Middlesex HA1 3UJ (Tel: 081-869 3312).

\section{Corrections}

F Clin Pathol 1994;47:205-8; Tillyer et al. The title of the correspondence should read "zinc protoporphyrin assays in patients with $a$ and $\beta$ thalassaemia trait." The title at present implies that zinc assays were performed which was not the case.

In paragraph 2 , the second sentence should read ". . . not only were the drugs causing substantial interference extremely unlikely in the outpatient and general practice population we studied, ...". Paragraph 3 second sentence should read "...Paul and Brumfitt's is $15 \mu \mathrm{mol} / \mathrm{mol}$ haem lower."

DR ML TILLYER

Estimation of haemoglobin concentrations using spectrophotometric tests. $\mathcal{f}$ Clin Pathol 1994;47:681.

The name of the author was given incorrectly as J Larner rather than AJ Larner. ANDREW J LARNER

f Clin Pathol 1993;46:1116-9. (Darjee R, Gibb AP. Serological Investigation into the association between Streptococcus bovis and colonic cancer.) The methods section refers to "NCTC10449 (Enterococcus fecalis), but this should read ATCC19433 (Enterococcus fecalis). NCTC10449 is in fact the reference number of the type strain of $S$ mutans.

AP GIBB

Increased pentane and carbon disulfide in the breath of patients with schizophrenia f Clin Pathol 1993;46:861-4. The concentrations of pentane and carbon disulfide were reported incorrectly. All values of pentane should be multiplied $\times 50$; all values of carbon disulfide $\times 0.05$. The statistical analyses and conclusions of the paper are not affected by these corrections.

MICHAEL PHILLIPS

Chu CM, Liaw YF. Coexpression of intercellular adhesion molecules and class I major histocompatibility compression antigens on hepatocyte membrane in chronic viral hepatitis. I Clin Pathol 1993;46: 1004-8. The correct version of fig $2 \mathrm{D}$ is reproduced below.

CHIA-MING CHU

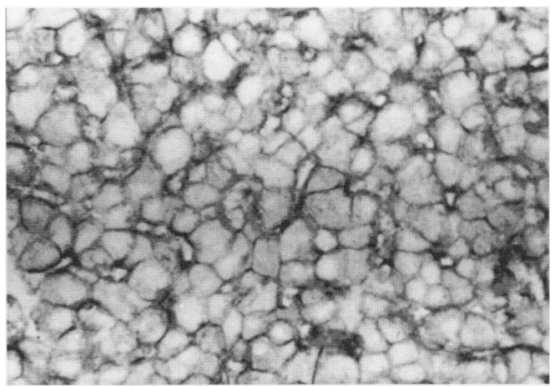

